\title{
A Need for Meaningful Visual Feedback of Lower Extremity Function After Stroke
}

\author{
Heather Thikey \\ Bioengineering \\ Department \\ University of Strathclyde \\ Glasgow, UK \\ heather.thikey@strath.ac. \\ uk
}

\author{
Dr. Frederike van Wjick \\ School of Health \\ Glasgow Caledonian \\ University, Glasgow, UK \\ Frederike.vanWijck@gcu \\ ac.uk
}

\author{
Prof. Madeleine Grealy \\ School of Psychological \\ Sciences and Health \\ University of Strathclyde \\ Glasgow, UK \\ m.grealy@strath.ac.uk
}

\author{
Prof. Philip Rowe \\ Bioengineering \\ Deptartment \\ University of Strathclyde \\ Glasgow, UK \\ philip.rowe@strath.ac.uk
}

\begin{abstract}
With the drive towards early supported discharge from the NHS, there is a need to support stroke survivors in the community with home-based rehabilitation. Computer games and virtual reality systems are increasing in popularity in the rehabilitation setting and seem encouraging in promoting exercise behaviour. However, in commercially available games, the quality of movement used to complete tasks can be commonly overlooked, where games are more focused on the end success of the task. Envisage gait rehabilitation is a study involving a rehabilitation aid that proposes to provide stroke survivors, and their therapists, with accurate visual feedback of their movement performance during gait-related exercises. This tool will provide users with meaningful visual feedback of not only the user's success but of the quality of their movements and their progress with time. This paper reviews current applications of computer games and virtual reality systems involving lower extremity function after stroke and the potential application of envisage gait rehabilitation in community and home-based stroke rehabilitation.
\end{abstract}

Keywords-community, function, gait, home, lower limb, rehabilitation, stroke, sub-acute, visual feedback

\section{INTRODUCTION}

Stroke is the third most common cause of death worldwide [1] and is the largest cause of complex disability in adults [2]. Two thirds of stroke survivors develop an abnormal walking pattern, with thirty percent still unable to walk independently six months later [3]. Walking problems after stroke can have a strong impact on an individual's independence in performing activities of daily living. Typical hemiplegic gait characteristics are slow walking speed, uneven step length, poor joint and posture control, and reduced weight bearing on the affected side $[4,5]$. Compensatory movements are often employed, such as 'hip hitching' to facilitate foot clearance in walking.

With an increasing ageing population, the prevalence of stroke, and its associated healthcare costs, is likely to escalate. Accordingly, the National Health Service has developed services that enable patients to be discharged earlier from hospital with rehabilitation support provided at home (early supported discharge or ESD). Reduction in the length of hospital stay has been reported by Langhorne et al. [6] to be an average of eight days shorter as a result of ESD and a further study by Anderson et al. [7] has suggested ESD to be a more cost-effective method than conventional hospital care.

Current evidence suggests that home-based rehabilitation is beneficial to stroke survivors [8]. By bringing rehabilitation services to the home setting, therapy can be adapted to each individual's environment and becomes more relevant to the patient's specific needs. The growing body of evidence-based studies supporting improved clinical outcomes as a result of ESD has been recognised in the Scottish Government's 'Better Heart Disease and Stroke Care Action Plan' [9]. One item on the agenda stated how rehabilitation should incorporate access to leisure centres for exercise. By facilitating gyms for the disabled, community-based rehabilitation and so improved community reintegration for the stroke survivor could be encouraged.

Current stroke rehabilitation guidelines recommend the intensive and repetitive practice of functional tasks after stroke [10]. However, for many patients, sheer repetition can be particularly unmotivating. One way of encouraging motivation is by providing patients with visual feedback of their movement in real-time or as offline feedback. Visual feedback gives patients knowledge of their movements and is known to promote movement re-education [11]. It can be particularly useful for proximal limb segments, which can be hard to see by the individual without contorting the body.

Visual feedback can take a variety of forms. Full-length mirrors are commonly used in clinical practice and can be used to train stroke survivors in, for example, maintaining trunk alignment during weight transference exercises. Video footage can also be used to give patients offline feedback of their walking performance. Yet the use of mirrors and video are not optimal after stroke since cognitive and self-image issues mean the patient can be distracted by their selfappearance. They are presented feedback as either a confusing mirror image or within a complicated visual field where their movement can be obscured by their clothing or the clinic background. An alternative form of visual feedback is displaying movement data in a numerical or pictorial form. A study by Lunenberger et al. [12] used line graphs and visual cueing with smiley-sad faces to indicate good or bad performance during gait training. Whether or not such feedback is meaningful to stroke patients is unclear. Although this kind of system tells the patient what is right and wrong, it does not implicitly instruct the patient how to adjust and correct their movements accordingly.

Computer games and virtual reality environments are leaders in the provision of engaging visual feedback. These systems, in particular, can provide patients with immersive visualisations that detract from the monotony of repetitive exercises. Use of this technology could potentially encourage and motivate stroke survivors to self-correct abnormal movement and prevent overreliance on therapists. 
The remainder of this paper is organised as follows. Section II gives a brief summary of computer games and virtual reality (VR) systems used in stroke rehabilitation and their limitations. Section III discusses the role of envisage gait rehabilitation in providing meaningful visual feedback. Section IV will discuss the future of envisage gait rehabilitation and a short summary of the paper will be presented in Section V.

\section{COMPUTER GAMES AND VIRTUAL REALITY IN LOWER EXTREMITY STROKE REHABILITATION}

The use of computer games and VR environments in stroke rehabilitation is increasing in popularity and results are encouraging that such techniques can enhance user engagement $[13,14]$. This section will briefly look at computer games and VR for lower extremity stroke rehabilitation.

Although not specifically designed for rehabilitation purposes, the Nintendo Wii is increasing in popularity for exercise after stroke and therapists have observed that the Wii games motivate patients in carrying out very repetitive movements [15]. The Nintendo Wii is primarily reported for its use with upper limb rehabilitation however it can also be used with games involving the lower limb. Despite recent enthusiasm, this technology has many limitations when considering stroke survivors and the use of such technology should be balanced with proof of efficacy [16]. Some games are too cognitively difficult and visually complex and, as a result, only the more able-bodied are able to make use of them. More importantly, quality of movement can be compromised in this system through use of different compensatory strategies. For example, in the Wii Boxing game, gamers can simulate a successful punch movement using simple flicks of the wrist rather than full arm extension and trunk movement [17].

Boian et al. [18] presented an example of gaming specifically aimed at lower extremity stroke rehabilitation. The participant's foot was strapped to a haptic robotic device that acted as a joystick with which the foot could control planes and speedboats during VR ankle-based exercises. Participants were able to see whether or not they had managed to successfully manoeuvre their vehicle in real-time. Another study, conducted by Jaffe et al. [19], superimposed virtual obstacles over video footage of the patient's lower body. This footage was displayed in realtime on a head mounted display and patients were instructed to step over the obstacles as they approached them. Results suggested that stepping over virtual objects was just as beneficial as stepping over real objects in shaping stepping characteristics. Moreover, participants mentioned the benefit of being able to view their movements in the saggital plane, where they were able to check their knee flexion and control their stepping.

VR has also been used to simulate community environments. Yang et al. [20] supported the use of gait training with simulated environments for improving community ambulation after stroke. Stroke survivors were asked to walk on a treadmill whilst facing projections of scenarios such as street crossings and paths with obstacles. This allowed users to engage in the repetitive practice of activities of daily living in a safe and controlled environment.
Current conventional clinical visual feedback is generally constricted to the use of mirrors. Although mirrors can be useful in providing patients with immediate feedback of their appearance, feedback is restricted to limiting viewpoints and is given as a confusing mirror image. Computer games and virtual reality methods provide a means of overcoming limitations associated with mirror feedback whilst encouraging motivation. Conversely, these applications are often focused on the success of tasks and important feedback on the user's strategy to complete a task can be overlooked.

A stroke rehabilitation aid should have therapeutic value and have the ability to accurately represent the user's movements. This will allow any compensatory movement strategies to be highlighted. Although user engagement is essential in a rehabilitation tool, it is important to also provide accurate and relevant biomechanical data that gives the user an indication of the quality of their movement. This is commonly referred to as knowledge of results (KR) and knowledge of performance (KP). KR relates to the outcome of the task whereas KP refers to the quality of the movement used to complete the task such as the speed to reach a target or the smoothness of the movement. In addition, to encourage the uptake of computer games and VR technology in rehabilitation practice, there is a need to promote awareness of their associated benefits through exposure and education. Despite the expansion of literature related to stroke rehabilitation, an evidence-to-practice gap in stroke still exists [21].

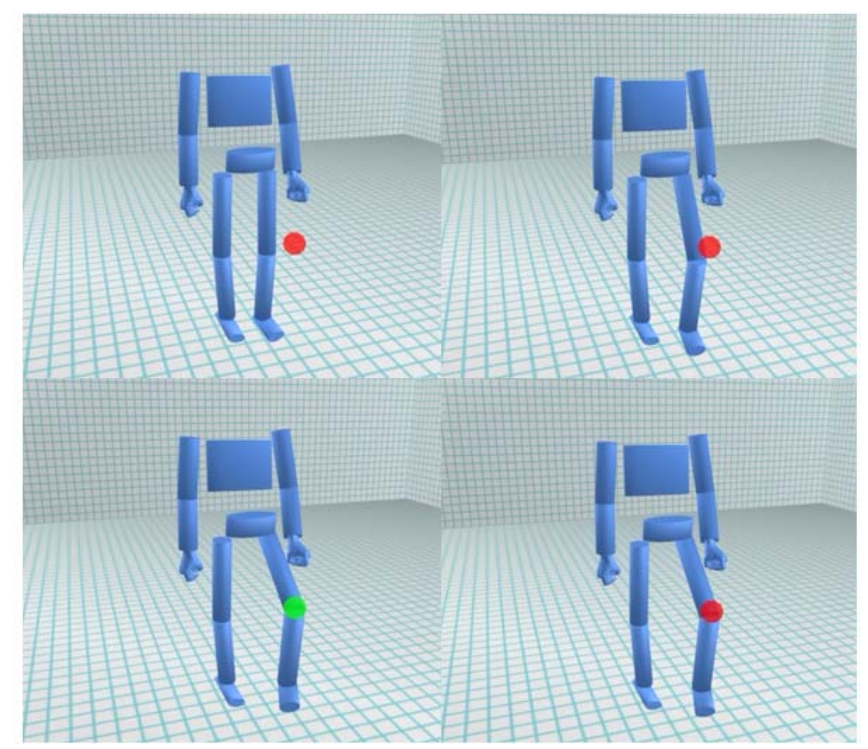

Figure 1. Virtual target-based knee lift exercise. Clockwise from top left: user lifts knee to reach red target, which turns green upon contact. Image courtesy of envisage team member Dr. David Loudon.

\section{ENVISAGE GAIT REHABILITATION}

Envisage is a cross-university project consisting of seven work packages, with a common goal of promoting independence through dynamic visualisation of biomechanical data. Work package four, envisage gait rehabilitation, is currently investigating the effect of the early provision of visual feedback with gait training after stroke. Envisage gait rehabilitation is a novel intervention strategy based on a previous study with healthy older adults. This work suggested that the envisage visualisations promoted discussion between different health professions and patients and gave users an increased understanding of 
the underlying biomechanics involved in everyday activities $[22,23]$.

The primary goal of envisage gait rehabilitation is to design a stroke rehabilitation aid that will provide users with meaningful visual feedback of KR and KP in movement performance. The proposed visual feedback is in the form of a computerised stick-like figure of the trunk, pelvis and lower limb segments. This computerised figure will mimic the movements of the user, providing a clear and depersonalised representation of the user's motion in realtime. To the authors' knowledge, no other visual feedback system in stroke rehabilitation has been presented in this way.

The theory of planned behaviour, as proposed by Ajzen [24], states that an individual's intentions can be predicted from their attitude towards behaviour, subjective norms and their perceived control over behaviour. It is anticipated that the envisage tool will promote an improved understanding of rehabilitation in stroke survivors and, in turn, an enhanced motivation and intention to practice. This could subsequently lead to an increase in exercise behaviour and, ultimately, enhanced mobility outcomes.

Movement data required by envisage gait rehabilitation is to be captured using small, Bluetooth-enabled motion sensors, which are currently being developed at Glasgow Caledonian University by Baillie et al. These will be attached to standardised positions on the user's trunk, pelvis and lower limb segments to feed real-time joint positions and angles into the visual feedback tool. The envisage tool requires only the input data of the position and orientation of joint centres, and consequently could be compatible with various different motion analysis systems.

Envisage gait rehabilitation comprises of three main functions:

- Providing patients with knowledge of their gait performance

- $\quad$ Providing patients with a pool of virtual therapeutic exercises for repetitive practise

- Providing patients with feedback of progress and therapists with a way of tracking progress

\section{A. KnOWledge of Gait Performance}

The tool will provide users with offline visual feedback of their gait performance (KP) using the stick-like figure described beforehand. The user will be able to zoom in, out and around, and have playback control of their representative stick-like figure. This will assist therapists in identifying and highlighting any gait impairments that are otherwise difficult to explain to the patient, such as range of motion and joint control, aiding communication between therapist and user.

\section{B. ViRTUAl Gait-RElated EXERCISES}

Users will be provided with a pool of virtual gait-related exercises involving targeted hip, knee and ankle movements and weight bearing in unilateral stance. The therapist will be able to select exercises suitable to the patient's specific requirements and adjust the level of difficulty of the task accordingly. These exercises will range from a breakdown of components of gait to more dynamic exercises such as stepping over and avoiding virtual obstacles. Figure 1 shows an example of a virtual exercise involving knee lifts. Here, the user lifts their knee to a virtual target which changes colour to indicate when it has been reached. The patient will be instructed on how to correctly execute their chosen task and the task's importance will be stressed by providing functional examples. For instance, a functional example of the knee lift exercise is clearing a step when walking up the stairs. Repetitive practice of such functional tasks will encourage transference of the exercises to everyday gaitrelated activities.

It is relatively straightforward for patients to recognise if they have managed to successfully hit a target (KR). However, patients may be less aware of the quality of their movements used to complete the task. Envisage gait rehabilitation will provide KP through parameters such as the average speed taken to hit a target, the range of motion achieved and coordination between body segments. The envisage tool will also be able to prompt awareness of any compensatory strategies employed as the user exercises.

\section{KNOWLEDGE of PROGRESSION}

A systematic review of stroke-related qualitative studies [25] identified a need to establish appropriate methods of measuring progress throughout rehabilitation. Envisage gait rehabilitation aims to provide knowledge of progress visually, using simple user-friendly visualisations. A typical characteristic of hemiparetic gait is uneven step length. Figure 2 shows an example of how step lengths can be communicated using visualisations. The shaded green box illustrates introducing a goal to reach, for which future attempts could be targeted. Figure 3 shows an example of how progress over time could be visualised. The tool will also explore the effects of communicating changes in other walking characteristics such as speed, range of motion and smoothness of gait.

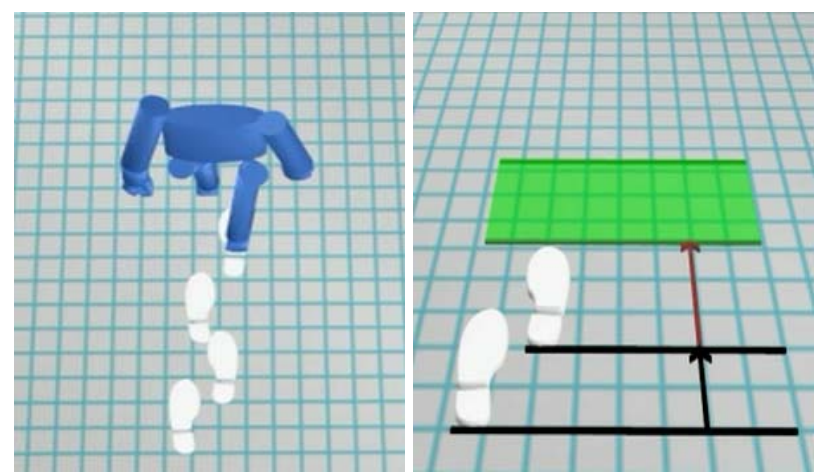

Figure 2. Visualising step lengths (left) and introducing targets (right). Images courtesy of Dr. David Loudon.

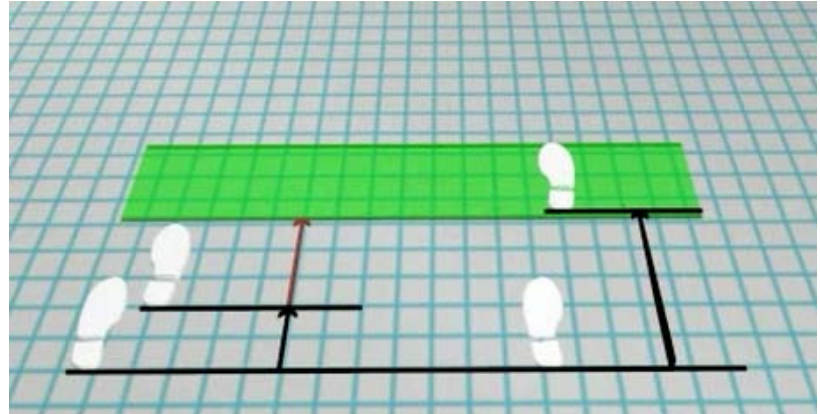

Figure 3. Communicating progress through visualisations of step length over time. Images courtesy of Dr. David Loudon.

Envisage gait rehabilitation will be capable of automatically logging session characteristics. This will 
allow users to monitor their progress over time. Furthermore, past scores could be used to benchmark future attempts for a more performance-orientated session. Encouraging motivation after stroke is essential, especially for users with low motivation, who are less likely to understand the purpose of the rehabilitation tasks as set by their therapists [26].

\section{Future of ENVISAGE GAIT REHABILITATION}

A single blind randomised controlled trial $(\mathrm{N}=45)$ will commence this year to investigate the effects of the early provision of the envisage visual feedback on mobility outcomes after stroke. The trial will be based in NHS hospitals and community centres in Lanarkshire. Stroke survivors in the sub-acute phase of stroke, who exhibit an abnormal gait pattern and have a Functional Ambulation Category score of one or more, will be eligible for this trial. Participants will be randomised into one of three groups: standard care only; gait training; and gait training with the proposed envisage visual feedback. Primary outcomes will look at temporal and spatial kinematics associated with walking and assessments of activities of daily living. These will be measured at baseline, six weeks and six months after randomisation. Qualitative measures will also be analysed to investigate any changes motivation and understanding in rehabilitation. It is anticipated that the results from this study will be used to power a future larger scale study, which could further analyse the effect of visual feedback on functional outcome after stroke and its effect on motivation and adherence in rehabilitation.

Although this particular study will be investigated in clinic and community-based settings, there is potential for the tool to be applied as a home-based system. As technology advances, small motion analysis systems for home-based rehabilitation are looking more and more within reach. Stroke survivors would then be able to engage in the repetitive practice of functional, therapeutic exercises as much as they want, when they want. Furthermore, the automatic logging of the envisage therapy tool would allow both patients and therapists to keep track of progress throughout the rehabilitation process and interact through telemedicine applications.

\section{SUMMARY}

A number of VR and computer game systems exist that incorporate visual feedback of lower extremity function. Many systems aim to increase motivation and the success of a task. However, it is of equal importance to consider the quality of the movement used to complete a task - especially in people with motor impairment due to stroke. For instance, an individual may be able to successfully step over an object, but they are not moving safely or efficiently if the success of the task comes from substituting hip and knee flexion with excessive hip elevation. Although games encourage motivation and adherence, they need to be taskrelated and of therapeutic value to ensure that they translate into everyday activities.

Envisage gait rehabilitation has the ability to accurately show the user how they move in real-time, or post-hoc, whilst exercising. The tool will provide taskdriven therapeutic exercises specific to stroke rehabilitation. Envisage gait rehabilitation aims to provide simple yet meaningful visual feedback of lower extremity function during stroke rehabilitation. It is envisioned that such feedback will result in enhanced mobility outcomes.

\section{ACKNOWLEDGMENT}

Heather Thikey wishes to acknowledge support from academic supervisors Prof. Philip Rowe, Prof. Madeleine Grealy and Dr. Frederike van Wijck. Special thanks to Dr. David Loudon for the provision of all images of visualisations and to all the users and members of the envisage reference group. The research of Heather Thikey has been supported by the Medical Research Council Lifelong Health and Wellbeing (LLHW) cross-council research programme (G0900583 Grant ID 91021).

\section{REFERENCES}

[1] J. Mackay, et al., The Atlas of Heart Disease and Stroke. World Health Organization (WHO), 2004. Available from: http://www.who.int/cardiovascular_diseases/resources/atlas/en I (Accessed $1^{\text {st }}$ December 2010).

[2] J. Adamson, Is stroke the most common cause of disability? Journal of Stroke and Cerebrovascular Diseases, 2004. 13:p. 171-177.

[3] R. A. States, E. Pappas, and Y. Salem, Overground physical therapy gait training for chronic stroke patients with mobility deficits (Review). The Cochrane Library, 2009(3).

[4] R. Dickstein, A. Dunsky, and E. Marcovitz, Motor imagery for gait rehabilitation in post-stroke hemiparesis. Physical Therapy, 2004. 84(12): p. 1167-1177.

[5] A. Pizzi, et al., Gait in hemiplegia: evaluation of clinical features with the Wisconsin gait scale. J Rehabil Med, 2007. 39:p. 170-174.

[6] P. Langhorne, et al., Early supported discharge service for stroke patients: a meta-analysis of individual patients' data. Lancet, 2005. 365: p. 501-06.

[7] C. Anderson, et al., Home or hospital for stroke rehabilitation? Results of a randomized controlled trial, I: Health outcomes at six months. Stroke, 2000. Vol. 31, pp1024-1031.

[8] L. Koch, L. H. Holmqvist, A. W. Wottrich, K. Tham, J. Pedro-Cuesta, Rehabilitation at home after stroke: a descriptive study of an individualized intervention. Clinical Rehabilitation, 2000. 14: p. 574583.

[9] The Scottish Government, Better heart disease and stroke care action plan. 2009: Edinburgh.

[10] Scottish Intercollegiate Guidelines Network 118. Management of patients with stroke: rehabilitation, prevention and management of complications, and discharge planning (A national clinical guideline), 2010 .

[11] C. Sackley, and N. Lincoln, Single blind randomized controlled trial of visual feedback after stroke: effects on stance symmetry and function. Disability \& Rehabilitation, 1997. 19(12): p. 536-546.

[12] L. Lunenburger, G. Colombo, and R. Riener, Biofeedback for robotic gait rehabilitation. Journal of NeuroEngineering and Rehabilitation, 2007. 4(1).

[13] H. Sveistrup, Motor rehabilitation using virtual reality. Journal of NeuroEngineering and Rehabilitation, 2004. 1: p. 10.

[14] J. W. Burke, et al., Optimising engagement for stroke rehabilitation using serious games. Vis Comput, 2009. 25: p. 1085-1099.

[15] A. Ramchandani, K. Carroll, R. Buenaventura, J. Douglas, and J. Liu, Wii-habilitation increases participation in therapy, IEEE confenernce of Virtual Rehabilitation, 2008, p. 69.

[16] J. Deutsch, et al. Wii-based compare to standard of care balance and mobility rehabilitation for two individuals post-stroke. IEEE. 2009.

[17] M. Pasch, N. Bianchi-Berthouze, B. Dijk, A. Nijholt, Movementbased sports video games: investigating motivation and gaming experience. Entertainment Computing, 2009. 1: p. 49-61.

[18] R. F. Boian, J. E. Deutsch, C. S. Lee, G. C. Burdea, J. Lewis, "Haptic Effects for Virtual Reality-Based Post-Stroke Rehabilitation," haptics, pp.247, 11th Symposium on Haptic Interfaces for Virtual Environment and Teleoperator Systems (HAPTICS'03), 2003

[19] D. Jaffe, D. Brown, C. Pierson-Carey, E. Buckley, H. Lew, Stepping over obstacles to improve walking in individuals with poststroke hemiplegia. Journal of Rehabilitation Research \& Development, 2004. 41: p. $283-292$. 
[20] Y. Yang, M. P. Tsai, T. Y. Chuang, W. H. Sung, R. Y. Wang, Virtual reality-based training improves community ambulation in individuals with stroke: a randomized controlled trial. Gait \& Posture, 2008. 28: p. 201-206.

[21] B. M. Demaerschalk, Evidence-based clinical practice education in cerebrovascular disease. Stroke, 2004. 35: p. 392-396.

[22] A. Macdonald, et al., Towards a design tool for visualizing the functional demand placed on older adults by everyday living tasks. Univ Access Inf Soc, 2007. 6: p. 137-144.
[23] A. Macdonald and D. Loudon (2009) Innovation in envisioning dynamic biomechanical data to inform healthcare and design guidelines and strategy. New dynamics of ageing programme

[24] I. Ajzen, The theory of planned behavior. Organizational Behavior and Human Decision Processes, 1991. 50: p. 179-211.

[25] C. McKevitt, et al., Qualitative Studies of Stroke: a systematic review. Stroke, 2004. 35: p. 1499-1505.

[26] N. Maclean, P. Pound, C. Wolfe, and A. Rudd, The concept of patient motivation: a qualitative analysis of stroke professionals' attitudes. Stroke, 2002. 33: p. 444-448. 